\title{
COPD-Disability-Index (CDI) - ein neues Verfahren zur Erfassung der COPD-bedingten Beeinträchtigung
}

\section{COPD Disability Index (CDI) - A New Instrument to Assess COPD-Related Disability}

\author{
Autoren \\ K. Kühl', W. Schürmann², W. Rief' \\ Institute \\ Universität Marburg \\ 2 Pneumologische Praxis Marburg
}

eingereicht 28.8.2008

akzeptiert nach Revision

21. 10. 2008

\section{Bibliografie}

Dol 10.1055/s-0028-1100829 Online-Publikation: 28.11.2008

Pneumologie 2009; 63: 136-143

(c) Georg Thieme Verlag KG

Stuttgart · New York

ISSN 0934-8387

\section{Korrespondenzadresse}

\section{Dipl. Psych. Kerstin Kühl}

Philipps-Universität Marburg, Fachbereich Psychologie, AG Klinische Psychologie und Psychotherapie Gutenbergstraße 18 35032 Marburg kuehl@staff.uni-marburg.de

\section{Zusammenfassung \\ $\nabla$}

Ziel der vorliegenden Studie ist die Evaluation einer neuen Skala zur Erfassung der COPD-bedingten Beeinträchtigung (CDI). Es handelt sich um die Adaptation des Pain-Disability-Index (PDI), der seit vielen Jahren zur Standarddiagnostik chronischer Schmerz-Patienten gehört. Der Grad der COPD-bedingten Beeinträchtigung wird auf einer 11-stufigen Ratingskala für 7 Lebensbereiche angegeben. An der Evaluationsstudie nahmen 143 COPD-Patienten (GOLD Stadium II - IV) und 105 Angehörige teil. Eine Hauptachsenanalyse ergab eine 1-Faktorenlösung, die 63,4\% der gemeinsamen Varianz aufklärt. Die Skala zeigt eine gute interne Konsistenz (Patienten-Selbsteinschätzung: Cronbachs alpha $=0,92$, AngehörigenFremdeinschätzung: Cronbachs alpha=0,91). Hinweise auf die Validität ergeben sich aus den hohen, signifikanten Korrelationen mit konstruktnahen Fragebogen [St. George's Respiratory Questionnaire (SGRQ): Pearsons Korrelationskoeffizient $r=0,59-0,83, p \leq 0,01$; European Quality of Life Questionnaire (EuroQol): $r=-0,59$, $\mathrm{p} \leq 0,01$ ] bzw. funktionellen Parametern (ShuttleWalk-Strecke: $r=-0,47 \mathrm{p} \leq 0,01 ; \mathrm{FEV}_{1}: \mathrm{r}=-0,26$, $\mathrm{p} \leq 0,01$ ) sowie niedrigen bzw. fehlenden Korrelationen mit konstruktfernen Fragebogen [Soziale Unterstützung (F-SozU): $\mathrm{r}=-0,09$, soziale Belastung (F-SozU): $r=0,17, p \leq 0,05$, Partnerschaftszufriedenheit: $r=-0,10]$. Beim COPD-Disability-Index handelt es sich um ein kurzes, einfach zu handhabendes, reliables und valides Instrument zur Erfassung der COPD-bedingten Beeinträchtigung.

\section{Einleitung \\ $\nabla$}

Die chronisch obstruktive Lungenerkrankung (COPD) gehört in Deutschland mit einer Prävalenzrate von 8,7\% zu den häufigen chronischen Erkrankungen im Erwachsenenalter [1]. Speziell

\section{Abstract \\ $\nabla$}

The aim of the present study was to evaluate a new scale for assessing COPD-related disability. The scale is an adaptation of the pain disability index (PDI), which is a standard instrument in diagnosing disability in chronic pain patients. An 11 -point rating scale is used to assess disability in seven different areas of life. The present study comprised a sample of 143 COPD patients (GOLD II-IV) and 105 spouses. A principal axes factor analysis revealed a one-factor solution explaining $63.4 \%$ of the variance. In the present sample, the scale showed good internal consistency (patients' self-report: Cronbach's alpha $=0.92$, spouses' report: Cronbach's alpha=0.91). The scale further demonstrated high correlations with questionnaires assessing related constructs [St. George's Respiratory Questionnaire (SGRQ): Pearson's correlation coefficients $r=0.59$ to 0.83 , $\mathrm{p} \leq 0.01$; European Quality of Life Questionnaire (EuroQol): $\mathrm{r}=-0.59, \mathrm{p} \leq 0.01$ ] and functional parameters (Shuttle-Walk-Distance: $r=-0.47$, $\mathrm{p} \leq 0.01 ; \mathrm{FEV}_{1} \%$ pred: $\mathrm{r}=-0.26, \mathrm{p} \leq 0.01$ ), as well as low correlations with questionnaires assessing distant constructs [social support (F-SozU): $r=-0.09$, social strain (F-SozU): $r=0.17, p \leq 0.05$, satisfaction with relationship: $r=-0.10$ ], suggesting good discriminant and convergent validity. The COPD disability index is a short, user-friendly, reliable and valid measure to assess COPD-related disability.

für die Altersgruppe der über 70-jährigen Männer liegt die Prävalenzrate sogar bei 19\% [1], was in Anbetracht des demographischen Wandels die Relevanz der Erkrankung hervorhebt. Die COPDErkrankung steht hierzulande bereits an 7. Stelle aller Todesursachen - Tendenz weiter steigend 
[2]. Gleichzeitig wird nicht nur die Anzahl der Jahre, die durch vorzeitigen Tod verloren gehen, sondern auch die Anzahl der Jahre, die mit COPD-bedingter Behinderung gelebt werden, weiter zunehmen. Aktuell ist davon auszugehen, dass die COPD-Erkrankung bis 2020, gemessen an den „disability-adjusted life years“ (DALY), auf den 5. Rang aller Erkrankungen vorrücken wird [3].

„Disability“ beschreibt das Ausmaß der subjektiv erlebten, krankheitsbedingten Einschränkungen bei Alltagsaktivitäten und Teilhabe an verschiedenen Lebensbereichen (z.B. Beruf, Selbstversorgung, etc.) im Sinne des bio-psycho-sozialen Gesundheitsbegriffs der Weltgesundheitsorganisation. Da die subjektive COPD-bedingte Beeinträchtigung nur einen geringen Zusammenhang zum medizinischen Schweregrad, d. h. zu objektivierbaren Abweichungen von einer vorgegebenen biomedizinischen Bezugsnorm zeigt, wird der Erfassung der subjektiven Beeinträchtigung zunehmend ein höherer Stellenwert beigemessen. Die Patientensicht hat in den letzten Jahren entsprechend auch in der Erfolgsbeurteilung unterschiedlicher Behandlungsansätze [4] oder bei der Indikationsstellung z. B. für Rehabilitationsmaßnahmen [5] wachsende Bedeutung erlangt. Einige Autoren befürworten sogar die Hinzuziehung der krankheitsbedingten Beeinträchtigung zur Klassifikation des COPD-Schweregrads [6]. Entsprechend wurden in den letzten Jahren mehrere Verfahren zur Einschätzung der Lebensqualität entwickelt. Die Datenbanken der American Thoracic Society (www.atsqol.org) oder die „Patient-Reported Outcome and Quality of Life Instruments“ (www.proqolid.org) listen 8 englischsprachige Verfahren zur Erfassung der krankheitsspezifischen Lebensqualität bei COPD-Patienten auf. Allerdings dominieren - auch im deutschsprachigen Raum - vor allem 2 multidimensionale Verfahren die Forschung: der Chronic Respiratory Questionnaire (CRQ) $[7,8]$ und der St. George Respiratory Questionnaire (SGRQ) $[9,10]$. Beide Fragebogen zeichnen sich durch vergleichbare, zufriedenstellende psychometrische Kennwerte in Bezug auf Reliabilität und Validität aus [11,12], benötigen jedoch aufgrund ihrer Länge zwischen 15 und 20 Minuten Durchführungszeit und konnten sich in der alltäglichen pneumologischen Praxis nicht durchsetzen.

Der CRQ beinhaltet 4 Subskalen (Kurzatmigkeit, Ermüdbarkeit, Stimmung, Krankheitsbewältigung), die sich faktorenanalytisch jedoch nicht bestätigen ließen [11]. Bei der längsten Subskala „Stimmung“ mit 7 von 20 Items wird durch die Instruktion kein eindeutiger Bezug zur Erkrankung hergestellt, so dass letztlich nicht beurteilbar ist, ob berichtete negative Affekte auf die Erkrankung zurückzuführen sind oder von dieser möglicherweise unabhängig vorhanden sind [13]. Hinzu kommen wechselnde Bezeichnungen der Rating-Abstufungen, was nicht nur eine gewisse kognitive Flexibilität voraussetzt, sondern auch zur Länge des Verfahrens (ca. 10 Seiten für 20 Items) beiträgt. Die Vergleichbarkeit zwischen verschiedenen Studien wird durch die vorhandenen 4 Versionen (Fragebogen vs. Interview-basiert, jeweils individualisiert oder standardisiert) erschwert.

Das zweite gängige Verfahren, der SGRQ weist einige Schwächen in der Praktikabilität auf. Die notwendige Gewichtung der einzelnen Items erschwert den Untersuchern die Auswertung. Die innerhalb der 50 Items mehrfach wechselnden Antwortformate (dichotome Antwortmöglichkeiten, 3-stufige, 4-stufige oder 5-stufige Ratingskalen), die wechselnden Bezeichnungen der Abstufungen oder deren wechselnde optische Anordnung erschweren den Patienten das Ausfüllen [13,14]. Hinzu kommen negative Formulierungen, die bei älteren Personen oder Personen mit niedrigerem Bildungsgrad zu Verwirrung führen kön- nen [14]. Das kann u.U. Hilfestellung durch Dritte erfordern, welche wiederum das Testergebnis beeinflussen kann [15].

Bei einer Patientengruppe, die bei Erreichung der GOLD Stadien III und IV in der Regel bereits ein höheres Lebensalter (> 65 Jahre) erreicht hat und bei der zu den zu erwartenden altersassoziierten kognitiven Veränderungen unter Umständen hyperkapnische [16] und hypoxische Schädigungen hinzukommen [17 - 19], sollte neben den multidimensionalen Verfahren auch ein besonders einfaches Verfahren zur Verfügung stehen, um die krankheitsspezifische Beeinträchtigung erfassen zu können. Das Verfahren sollte möglichst wenig kognitive Anforderungen an die Patienten stellen, damit die COPD-bedingte Beeinträchtigung auch bei schwer kranken, hoch betagten oder kognitiv leicht eingeschränkten Patienten erfasst werden kann.

Zur Erfassung der schmerzbedingten Beeinträchtigung gibt es ein solches Instrument: den Pain-Disability-Index (PDI) [20], deutsche Version [21,22]. Er ist mit 7 Items ein sehr kurzes und zeitökonomisches Verfahren, welches trotz seiner Kürze über verschiedene Studien hinweg mit einem Cronbachs alpha von .88 und einer mittleren Trennschärfe der Items von .67 gute Gütekriterien aufweist [21]. Faktorenanalysen ergeben zumeist eine 1-Faktoren-Lösung ([21,23-25]; abgesehen von [26]). Verschiedene Untersuchungen belegen übereinstimmend sowohl die Reliabilität als auch die Validität der Skala [21]. Der PDI gehört daher seit vielen Jahren in Deutschland auf Empfehlung der Deutschen Gesellschaft zum Studium des Schmerzes (DGSS) zur Standarddiagnostik bei chronischen Schmerz-Patienten [27].

Ziel der vorliegenden Studie war es, die biometrischen Eigenschaften der neuen Skala zur Erfassung der COPD-bedingten Beeinträchtigung (CDI) zu untersuchen und zu prüfen, ob mit dem CDI die krankheitsbedingte Beeinträchtigung bei COPD-Patienten erfasst werden kann. Zur Validierung werden Vergleiche mit konstruktnahen (allgemeine und krankheitsspezifische Lebensqualität, Psychopathologie, Fremdeinschätzung durch Angehörige, Gehstrecke und Einsekundenkapazität) und konstruktfernen Maßen (Soziale Unterstützung/Belastung und Partnerschaftszufriedenheit) berechnet.

\section{Methode \\ $\nabla$}

In einer pneumologischen Praxis (W. Schürmann) wurden über einen Zeitraum von 9 Monaten alle COPD-Patienten, in den GOLD Stadien II-IV konsekutiv in die Studie eingeschlossen. Ausschlusskriterien waren eine Exazerbation innerhalb des letzten Monats oder schwere akute Erkrankungen (z. B. Myokardinfarkt, Tumore etc.) innerhalb des letzten Jahres bei sich oder den Partnern/innen, um die COPD-bedingte Beeinträchtigung im stabilen Krankheitszustand erfassen zu können. In der Praxis wurde die Lungenfunktionsmessung mit einem "MasterScreenBody“ der Firma Viasys/Jaeger nach den ATS-Richtlinien sowie der Shuttle-Walk-Test (SWT) als Gehtest $[28,29]$ durchgeführt. 3-10 Tage später wurde den Patienten und Angehörigen zu Hause die Fragebogenbatterie vorgegeben. Die Untersucherin (K. Kühl) war während der gesamten Zeit anwesend, um Verständnisfragen beantworten zu können. Alle Teilnehmer der Studie erhielten eine Aufwandsentschädigung von 10,-€.

Verwendete Messinstrumente:

- COPD-Disability-Index (CDI): Die Originalversion des Fragebogens besteht aus 7 Items (Einschränkungen in den Bereichen: familiäre und häusliche Verpflichtungen, Erholung, soziale Aktivitäten, Beruf, Sexualleben, Selbstversorgung und 
lebensnotwendige Tätigkeiten wie essen, schlafen und atmen). In der vorliegenden Studie wurde das Item 7 auf die Beeinträchtigung der Atmung begrenzt. Wie in der Originalversion soll die COPD-bedingte Beeinträchtigung anhand einer 11-stufigen Ratingskala von „0= keine Beeinträchtigung“ bis „10= völlige Beeinträchtigung“ in den einzelnen Lebensbereichen angegeben werden. Der CDI wurde jeweils in einer Selbst- und einer Fremdbeurteilungsversion vorgegeben. Die Patienten wurden zunächst instruiert, in einer Selbsteinschätzung ihre eigene Beeinträchtigung anzugeben $\left(\mathrm{P}-\mathrm{CDI}^{\mathrm{Se}}\right)$. Anschließend wurden sie instruiert, ein Fremdrating abzugeben. Das heißt, sie sollten einschätzen, wie stark aus ihrer Sicht die Angehörigen durch die COPD-Erkrankung beeinträchtigt sind $\left(\mathrm{P}-\mathrm{CDI}^{\mathrm{Fe}}\right)^{1}$. Den Angehörigen wurde der CDI ebenfalls mit den 2 Instruktionen vorgegeben, so dass von ihnen auch eine Selbsteinschätzung (Beeinträchtigung durch den kranken Partner: $\left.\mathrm{A}-\mathrm{CDI}^{\mathrm{Se}}\right)$ und eine Fremdeinschätzung der COPD-bedingten Beeinträchtigung des Patienten (A-CDI ${ }^{\mathrm{Fe}}$ ) vorliegt.

- St. George's Respiratory Questionnaire (SGRQ): Die deutsche Version des SGRQ $[10,30]$ erfasst mit 50 Items über 3 Subskalen („Symptome“, „Aktivitäten“, „Belastung“) die krankheitsspezifische Lebensqualität. Zusätzlich kann ein Gesamtwert berechnet werden. Niedrige Werte spiegeln eine geringe bzw. hohe Werte eine hohe krankheitsbedingte Einschränkung der Lebensqualität wider.

- European Quality of Life Questionnaire (EuroQoL): Der EuroQoL [31] erfasst mithilfe einer visuellen Analogskala den subjektiven Gesundheitszustand als Maß der allgemeinen gesundheitsbezogenen Lebensqualität. Die Skala reicht von „0= der schlechteste vorstellbare Gesundheitszustand“ bis „100= der beste vorstellbare Gesundheitszustand“.

- Fragebogen zur sozialen Unterstützung (F-SozU): Zur Erfassung der subjektiv wahrgenommenen und erlebten sozialen Unterstützung wurde die F-SozU-Kurzform (22 Items) eingesetzt. Zur Erhebung der subjektiv wahrgenommenen und erlebten sozialen Belastung wurde die Subskala „Belastung“ (12 Items) aus der Langform des F-SozU verwandt [32].

- Symptom-Checklist-90-R(SCL-90-R): Die SCL-90-R [33] erfasst die subjektive Beeinträchtigung durch 90 körperliche und psychische Symptome innerhalb der letzten Woche. Es können u.a. die Subskalen „Depressivität“, „Ängstlichkeit“ und „Somatisierung“ berechnet werden.

- Partnerschaftszufriedenheit: Das Item „Wie glücklich schätzen Sie Ihre Partnerschaft im Augenblick ein?“ ( $0=$ sehr unglücklich - 5 sehr glücklich) wurde aus dem Partnerschaftsfragebogen (PFB) [34] entnommen.

\section{Auswertung \\ $\nabla$}

Die statistische Auswertung wurde mit SPSS (Version 15.0, SPSS Inc., Chicago, IL) vorgenommen. Nach der deskriptiven Beschreibung der Stichprobe wurde im Rahmen einer Itemanalyse die Verteilung überprüft, wurden Mittelwerte, Standardabweichung, Trennschärfekoeffizienten und Schwierigkeit der CDIItems berechnet. Anschließend wurde eine Hauptachsenanalyse (Variante einer Faktorenanalyse) durchgeführt. Zur Reliabilitätsbzw. Skalenanalyse wurden interne Konsistenz, Schiefe und

\footnotetext{
1 „P-...“: Angaben der Patienten, „A-...“: Angaben der Angehörigen, „... Se“: Selbstangaben, ,... Fe“": Fremdangaben
}

Kurtosis der Skala berechnet. Zur Bestimmung der konvergenten Validität wurden bivariate Korrelationen zwischen dem CDI und konstruktnahen Fragebogen sowie funktionellen Parametern berechnet. Zur Bestimmung der divergenten Validität wurden Korrelationen zu konstruktfernen Fragebogen berechnet. Zur Überprüfung etwaiger Mittelwertsunterschiede wurden univariate Varianzanalysen mit Tukey-Kontrasten und t-Tests berechnet.

\section{Patienten \\ $\nabla$}

Von 165 Patienten $^{2}$ lehnten nur 15 (9,1\%) eine Studienteilnahme ab. 10 Personen (7 Patienten [4,2\%]/3 Angehörige [2,7\%]) mussten im Nachhinein ausgeschlossen werden. Die Ausschlussgründe waren: Exazerbation (2) bzw. Tumordiagnosen (3) in der Zeit zwischen Praxis- und Hausbesuch, Psychose (1), zu viele fehlende Werte (1), fragliche demenzielle Erkrankung (2) oder unzureichende Deutschkenntnisse (1). Ein weiteres Paar wurde ausgeschlossen, da beide COPD-Patienten waren. Dadurch wurden 143 COPD-Patienten bzw. 105 Paare in die Auswertung ${ }^{3}$ einbezogen ( Tab. 1).

\section{Ergebnisse \\ $\nabla$}

Itemanalyse

Der Gesamtsummenscore des CDI und Item 4 sind normalverteilt, die übrigen Items sind nicht normalverteilt. Im Gegensatz zu vielen klinischen Fragebögen, die zumeist linkssteile Verteilung aufweisen, werden hier alle 11 Abstufungen genutzt. Das spiegelt sich entsprechend in den Angaben zur prozentualen Häufigkeit wider, mit der die 0 (,überhaupt nicht beeinträchtigt“) oder die 10 („völlig beeinträchtigt“) angekreuzt wurden (vgl. Tab.2). Erfreulich sind die ausgesprochen hohen Trennschärfen sowohl in der Selbstbeurteilungs- $\left(\mathrm{r}_{\mathrm{it}}: 0.66-0.83\right)$ als auch in der Fremdbeurteilungsversion durch die Angehörigen $\left(r_{i t}: 0.56-0.83\right)$. Die mittlere Interitemkorrelation beträgt 0.63 . Die einzelnen Items korrelieren zwischen 0.49-0.77. Das Item „Beeinträchtigung der Atmung“ weist im Vergleich zu den übrigens Items die niedrigsten Interitemkorrelationen auf (zwischen 0.49-0.63). Der Anteil fehlender Werte ist mit 0,14\% außerordentlich gering, alle Auslassungen bezogen sich auf die COPDbedingte Beeinträchtigung des Sexuallebens.

Der CDI-Gesamtwert wird über alle 7 Items summiert (Maximum 70). Unbezahlte Tätigkeiten wie Hausfrauentätigkeit werden als Beruf gewertet. Wenn auch eine solche Tätigkeit verneint wird, geht das Item 1 zweifach in den CDI-Gesamtwert ein.

\section{Faktorenanalyse}

Vor der faktorenanalytischen Auswertung wurde zunächst mithilfe des Kaiser-Meyer-Olkin-Kriteriums (KMO) überprüft, ob alle Variablen ausreichend miteinander korrelieren. Die Stichprobe weist einen hohen KMO-Koeffizienten (0.91) auf. Zudem kann nach dem Bartlett-Test $\left(\chi^{2}=688402 ; p \leq 0,001\right)$ davon ausgegangen werden, dass auch in der Grundgesamtheit von 0 verschiedene Interkorrelationen auftreten. Eine faktorenanalyti-

\footnotetext{
${ }^{2}$ Im Weiteren wird zur Wahrung des Leseflusses die Form „Patienten“ für Patienten und Patientinnen bzw. der Begriff „Angehörige“ für Ehefrauen und Ehemänner verwendet.

${ }^{3}$ Erste Ergebnisse wurden auf dem 49. Kongress der Deutschen Gesellschaft für Pneumologie und Beatmungsmedizin (DGP) im April 2008 in Lübeck vorgestellt.
} 


\begin{tabular}{|c|c|c|c|}
\hline & Patienten $(n=143)$ & & Angehörige $(n=105)$ \\
\hline Alter (Jahre) & $\begin{array}{l}67,0 \pm 9,5 \\
\min : 40 \\
\max : 90\end{array}$ & & $\begin{array}{l}64,0 \pm 9,3 \\
\min : 43 \\
\max : 84\end{array}$ \\
\hline Geschlecht & $\begin{array}{l}31 \mathrm{~F}: 112 \mathrm{M} \\
(21,7 \%: 78,3 \%)\end{array}$ & & $\begin{array}{l}86 \mathrm{~F}: 19 \mathrm{M} \\
(81,9 \%: 18,1 \%)\end{array}$ \\
\hline \multicolumn{4}{|l|}{ Familienstand } \\
\hline ledig & $8(5,5 \%)$ & & \\
\hline getrennt & $11(7,7 \%)$ & & \\
\hline verwitwet & $14(9,8 \%)$ & & \\
\hline verheiratet & $110(76,9 \%)$ & & $105(100 \%)$ \\
\hline Ehedauer (Jahre) & & $\begin{array}{l}37,8 \pm 12,8 \\
\min : 4 \\
\max : 66\end{array}$ & \\
\hline \multicolumn{4}{|l|}{ Schulbildung } \\
\hline Volks-/Hauptschule & $110(76,9 \%)$ & & $86(80,1 \%)$ \\
\hline Realschule & $19(13,3 \%)$ & & $14(9,8 \%)$ \\
\hline Abitur & $14(9,8 \%)$ & & $5(3,5 \%)$ \\
\hline \multicolumn{4}{|l|}{ Berufstätigkeit/Rente } \\
\hline berufstätig & $28(19,6 \%)$ & & $35(24,5 \%)$ \\
\hline Frührente & $69(48,3 \%)$ & & $25(17,5 \%)$ \\
\hline Rente & $46(32,2 \%)$ & & $45(31,5 \%)$ \\
\hline GOLD Stadien & $\mathrm{FEV}_{1}$ in \% des Solls & SWT & \\
\hline II $n=68(47,6 \%)$ & $62,9 \pm 9,6$ & $402 \pm 241$ & \\
\hline III n= $62(43,4 \%)$ & $40,8 \pm 5,3$ & $219 \pm 161$ & \\
\hline $\operatorname{IV} n=13(9,1 \%)$ & $26,4 \pm 2,8$ & $118 \pm 133$ & \\
\hline
\end{tabular}

Tab. 1 Stichprobenbeschreibung.

$\mathrm{FEV}_{1}$ : Einsekundenkapazität in Prozent des Solls; SWT: Shuttle Walking-Test (in Metern)

Tab.2 Item- und Skalenkennwerte des COPD-Disability-Index (CDI).

\begin{tabular}{|c|c|c|c|c|c|c|c|c|}
\hline \multirow[b]{2}{*}{ Itemkennwerte } & \multicolumn{4}{|l|}{$\mathrm{P}-\mathrm{CDI} \mathrm{Se}^{\mathrm{Se}}$} & \multicolumn{3}{|l|}{$\mathrm{A}-\mathrm{CDI} \mathrm{Fe}$} & Angehörige: $n=105$ \\
\hline & $\mathrm{MW}_{ \pm} \mathrm{SD}$ & $\mathrm{p} / 0$ & $\mathrm{p} / 10$ & $r_{i t}$ & $M W_{ \pm} S D$ & $r_{i t}$ & $\mathrm{p} / 0$ & $\mathrm{p} / 10$ \\
\hline 1 häusliche Verpflichtungen & $5,16 \pm 3,09$ & 8,4 & 13,3 & 0,82 & $5,26 \pm 3,63$ & 0,79 & 14,7 & 14,0 \\
\hline 2 Erholung & $5,55 \pm 3,24$ & 9,8 & 19,6 & 0,80 & $5,33 \pm 3,69$ & 0,79 & 15,4 & 73,4 \\
\hline 3 Soziale Aktivitäten & $4,22 \pm 3,55$ & 20,3 & 13,3 & 0,83 & $4,28 \pm 3,74$ & 0,83 & 22,4 & 8,4 \\
\hline 4 Beruf & $4,81 \pm 3,04$ & 14,0 & 11,9 & 0,66 & $5,01 \pm 3,64$ & 0,79 & 15,4 & 14,0 \\
\hline 5 Sexualität & $5,13 \pm 3,75$ & 19,6 & 22,4 & 0,73 & $5,18 \pm 3,72$ & 0,71 & 14,0 & 16,8 \\
\hline 6 Selbstversorgung & $2,68 \pm 3,41$ & 46,2 & 8,4 & 0,76 & $2,11 \pm 2,95$ & 0,62 & 40,6 & 0,7 \\
\hline 7 Atmung & $3,65 \pm 3,32$ & 26,6 & 7,0 & 0,70 & $3,96 \pm 3,61$ & 0,56 & 23,1 & 5,6 \\
\hline Skalenkennwerte & $\mathrm{MW}_{ \pm} \mathrm{SD}$ & \multicolumn{2}{|c|}{ Schiefe } & SDF & Kurtosis & \multicolumn{2}{|c|}{ Kurtosis Fehler } & $\alpha$ \\
\hline $\mathrm{P}-\mathrm{CDI}^{\mathrm{Se}}$ & $4,46 \pm 2,76$ & \multicolumn{2}{|c|}{0.19} & 0.20 & -0.99 & \multicolumn{2}{|c|}{0.40} & 0.92 \\
\hline $\mathrm{A}-\mathrm{CDI} \mathrm{Fe}^{\mathrm{Fe}}$ & $4,45 \pm 2,87$ & \multicolumn{2}{|c|}{0.00} & 0.24 & -1.14 & \multicolumn{2}{|c|}{0.47} & 0.91 \\
\hline
\end{tabular}

MW: Mittelwert; SD: Standardabweichung; p: Wahrscheinlichkeit, dass die Items 0 bzw. 10 bejaht wurden (Bandbreite $0-10) ; r_{i t}$ : korrigierte Trennschärfe; SDF: Standardfehler der Schiefe; $\alpha$ : Cronbachs alpha; P-CDI ${ }^{\mathrm{SE}}$ : Patienten-Selbsteinschätzung der COPD-bedingten Beeinträchtigung; A-CDIFE: Fremdeinschätzung der COPD-bedingten Beeinträchtigung der Patienten durch die Angehörigen

sche Auswertung ist demnach empfehlenswert [14]. Eine Hauptachsenanalyse (vgl. Tab.3) ergab unter Berücksichtigung des Eigenwertkriteriums größer 1 einen Faktor, der 63,4\% der Gesamtvarianz aufklärt.

Die 7 Items lassen sich somit gut zu einem Gesamtwert COPDbedingte Beeinträchtigung zusammenfassen. Ein hoher Wert spiegelt dabei ein hohes Ausmaß an subjektiver Beeinträchtigung wider (Maximum =70).

\section{Reliabilität}

Die interne Konsistenz als Reliabilitätsmaß ist mit 0.92 bemerkenswert hoch für die Kürze der Skala (vgl. $\bullet$ Tab.2). Die interne Konsistenz der Fremdbeurteilungsversion durch die Angehörigen ist ebenfalls sehr gut.

\section{Validität}

Konvergente und divergente Validitäten wurden bestimmt, indem der CDI mit konstruktverwandten Skalen (z. B. SGRQ, EuroQol) und funktionellen Parametern $\left(\mathrm{FEV}_{1}\right.$ in \% des Solls, Gehstrecke) bzw. konstruktfernen Skalen (F-SozU, Partnerschaftszufriedenheit) korreliert wurden (vgl. $\odot$ Tab.4). Die Korrelationen entsprechen dabei in Richtung und Höhe durchgängig den Erwartungen und belegen die konvergente und divergente Validität des CDI.

Korrelationskoeffizienten größer 0.60 zwischen Selbstbeurteilungsverfahren sprechen für eine hohe konvergente Validität $(<0.40=$ niedrig, $0.40-0.60=$ mittel; nach [35]). Diese Koeffizienten werden mit den SGRQ-Subskalen „Aktivitäten“ und „Belastung“ sowie dem SGRQ-Gesamtwert deutlich überschritten. Die SGRQ-Subskala „Symptome“ und die allgemeine gesundheitsbe- 


\begin{tabular}{cll}
\hline Tab.3 & Hauptachsenanalyse: Itemladungen des COPD-Disability-Index (CDI). \\
$\mathbf{N r}$. & Item-Inhalt & Faktor I \\
1 & familiäre und häusliche Verpflichtungen & 0.87 \\
\hline 2 & Erholung & 0.84 \\
\hline 3 & soziale Aktivitäten & 0.87 \\
\hline 4 & Beruf & 0.70 \\
\hline 5 & Sexualleben & 0.77 \\
\hline 6 & Selbstversorgung & 0.79 \\
\hline 7 & Atmung & 0.73 \\
\hline & & \\
\hline
\end{tabular}

Tab.4 Indikatoren konvergenter und divergenter Validität.

\begin{tabular}{|c|c|}
\hline Indikatoren & $\mathbf{r}$ \\
\hline \multicolumn{2}{|c|}{ Konvergente Korrelationen } \\
\hline \multicolumn{2}{|c|}{ Selbstbeurteilungen (Fragebögen) } \\
\hline SGRQ: Symptome & $0.59^{* *}$ \\
\hline SGRQ: Aktivitäten & $0.74^{* *}$ \\
\hline SGRQ: Belastung & $0.81^{* *}$ \\
\hline SGRQ: Gesamt & $0.83^{* *}$ \\
\hline EuroQol & $-0.59 * *$ \\
\hline SCL-90-R: Depressivität & $0.52^{* *}$ \\
\hline SCL-90-R: Ängstlichkeit & $0.47^{* *}$ \\
\hline SCL-90-R: Phobische Angst & $0.30 * *$ \\
\hline \multicolumn{2}{|c|}{ Fremdbeurteilung durch Ehepartner (Fragebögen) } \\
\hline $\mathrm{A}-\mathrm{CDI} \mathrm{Fe}^{\mathrm{Fe}}$ & $0.69^{* *}$ \\
\hline \multicolumn{2}{|l|}{ Funktionelle Parameter } \\
\hline $\mathrm{FEV}_{1}$ in $\%$ des Solls & $-0.26^{* *}$ \\
\hline SWT: Gehstrecke & $-0.47^{* *}$ \\
\hline \multicolumn{2}{|l|}{ Divergente Korrelationen } \\
\hline \multicolumn{2}{|c|}{ Selbstbeurteilungen (Fragebögen) } \\
\hline F-SozU: Soziale Unterstützung & -0.09 \\
\hline F-SozU: Soziale Belastung & $0.17^{*}$ \\
\hline Partnerschaftszufriedenheit & -0.10 \\
\hline \multicolumn{2}{|c|}{$\begin{array}{l}\text { r: Pearson Korrelationen; SGRQ: St. George's Respiratory Questionnaire; } \\
\text { EuroQol: European Quality of Life Questionnaire; SCL-90-R: Symptom } \\
\text { Checklist } 90-R ; A-C D I \text { Fe: Fremdeinschätzung der COPD-bedingten Beein- } \\
\text { trächtigung der Patienten durch die Angehörigen; FEV } 1 \text { : Einsekunden- } \\
\text { kapazität in Prozent des Solls; SWT: Shuttle Walking-Test (in Metern); } \\
\text { F-SozU: Fragebogen zur sozialen Unterstützung; *: } \mathrm{p} \leq 0,05=\text { signifikant, } \\
{ }^{* *}: \mathrm{p} \leq 0,01 \text { sehr signifikant }\end{array}$} \\
\hline
\end{tabular}

zogene Lebensqualität (EuroQol) verfehlen diesen Bereich nur knapp. CDI und SGRQ-Gesamtwert zeigen vergleichbare Korrelationen zu den Subskalen „Ängstlichkeit“ und „Depressivität“ der SCL-90-R (SGRQ-T und „Depressivität“: $r=0.59$, „Ängstlichkeit“: $r=0.53)$. Hervorzuheben ist die hohe Korrelation $(r=0.83)$ zwischen dem CDI und dem Gesamtwert des SGRQ, obwohl der CDI wesentlich kürzer (7 vs. 50 Items) ist als der SGRQ. Die Korrelationen zwischen dem CDI und den objektiven Parametern fallen - wie zu erwarten - im Vergleich zu den Selbstbeurteilungsverfahren niedriger aus, sind jedoch ebenfalls signifikant.

\section{Ergebnisse der Zusatzanalysen}

Eine univariate Varianzanalyse (vgl. $\odot$ Tab.5) ergab einen erwarteten signifikanten Mittelwertsunterschied beim CDI in Abhängigkeit vom COPD-Stadium $(\mathrm{F}(2 / 143)=4,87, \mathrm{p} \leq 0,001)$. Patienten mit GOLD Stadium II unterscheiden sich jeweils signifikant von den Patienten mit GOLD Stadium III und IV. Der Unterschied zwischen den GOLD-Stadien III und IV wurde dagegen nicht signifikant. Univariate Varianzanalysen und Tukey-Kontraste ergaben auch für den Gesamtwert (SGRQ: $F(2 / 143)=9,00, p \leq 0,001)$ sowie die 3 Subskalen des SGRQ (Symptome: $F(2 / 143)=5,49, p \leq 0,01$, Aktivitäten: $\mathrm{F}(2 / 143)=14,02, \mathrm{p} \leq 0,001$, Beeinträchtigung: $\mathrm{F}$ $(2 / 143)=6,08, p \leq 0,01)$ vergleichbare signifikante Mittelwertsunterschiede in Abhängigkeit vom COPD-Stadium.

Überraschend dagegen ist der Mittelwertsunterschied in Abhängigkeit vom Geschlecht. In der vorliegenden Stichprobe fühlen sich die COPD-Patientinnen signifikant weniger beeinträchtigt als die COPD-Patienten (Frauen: $\mathrm{n}=31, \mathrm{M}=3,10, \mathrm{SD}=2,99$; Männer: $n=112, M=4,83, S D=2,59 ; t=-3,18, d f=141 ; p \leq 0,05)$.

Betrachtet man die Einschätzungen der Paare zur COPD-bedingten Beeinträchtigung ergeben sich zwischen den Ehepartnern weder für die Patienten noch für die COPD-bedingte Beeinträchtigung der Angehörigen in den Selbst- bzw. Fremdbeurteilungen signifikante Mittelwertsunterschiede. Dies ist ein deutlicher Beleg für die Validität des Verfahrens.

Die hohe, signifikante Korrelation in den Selbstbeurteilungen der COPD-bedingten Beeinträchtigung zwischen Angehörigen $\left(\mathrm{A}-\mathrm{CDI}^{\mathrm{Se}}\right)$ und Patienten $\left(\mathrm{P}-\mathrm{CDI}^{\mathrm{Se}}\right)(\mathrm{r}=0.53, \mathrm{p} \leq 0,001)$ ist ein Beleg dafür, dass die Erkrankung nicht nur den Patienten, sondern auch die Partner belastet.

\section{Schlussfolgerungen \\ $\nabla$}

In der vorliegenden Arbeit wurde ein neues Instrument zur Erfassung der COPD-bedingten Beeinträchtigung bei Patienten mit chronisch obstruktiven Lungenerkrankungen vorgestellt. Mithilfe dieser eindimensionalen, 7-Item kurzen Skala lässt sich die krankheitsbedingte Beeinträchtigung ökonomisch, reliabel und valide erfassen.

Die Ergebnisse der psychometrischen Überprüfung sprechen für dieses praktikable Verfahren. Die Ausfüllzeit liegt bei zwei Minuten, die Auswertungszeit (Summenwertbildung) unter einer Minute. Alle Patienten konnten den Fragebogen ohne Verständnisfragen eigenständig ausfüllen. Dies spricht für die gute Verständlichkeit und Akzeptanz des Verfahrens auf Seiten der Patienten und spiegelt sich auch in dem äußerst geringen Anteil fehlender Werte $(0,14 \%)$ wider.

Die Hauptachsenanalyse bestätigt die Eindimensionalität des Konstrukts COPD-bedingte Beeinträchtigung, wie sie auch beim Pain-Disability-Index (PDI) zumeist gezeigt wurde [21,24,25]. Wenn in der Literatur teilweise auch von einer 2-Faktorenstruktur ausgegangen wird [36-38], geht das auf Tait u. Mitarb. zurück, die dies entgegen statistischer Konventionen bereits 1987 [23] postulierten und an einer großen Stichprobe von Rückenschmerzpatienten zeigen konnten [26]. Da unter biometrischen Gesichtspunkten eine Subskala mit 2 Items jedoch zweifelhaft erscheint, ist es erfreulich, dass der COPD-Disability-Index eindeutig eindimensional ist. Der aufgeklärte Varianzanteil von $63,4 \%$ liegt über den für den PDI berichteten aufgeklärten Varianzanteilen von $49-56 \%$ [25,24]. Alle Items laden hoch auf dem einen Faktor, welches ein Beleg für die Homogenität des Messinstruments ist.

Bei dem Item 7 „Beeinträchtigung der Atmung“ hätte man vermuten können, dass alle übrigen CDI-Items von diesem Item stark beeinflusst wären. Tatsächlich zeigt dieses Item aber im Vergleich zu den übrigen CDI-Items die niedrigsten Interitemkorrelationen, was dafür spricht, dass dieses Item einen eigenständigen Beitrag zur Skala leistet. Möglicherweise führte die kurze Erläuterung „dieser Bereich umfasst alle Probleme mit der Atmung“ dazu, dass die Patienten dieses Item nicht als Frage 
Tab.5 CDI- und SGRQ-Mittelwertunterschiede in Abhängigkeit vom COPD-Stadium.

\begin{tabular}{|c|c|c|c|c|c|c|}
\hline \multirow[t]{4}{*}{ Messinstrumente } & \multicolumn{3}{|l|}{ GOLD-Stadien } & \multicolumn{3}{|l|}{ Signifikanz } \\
\hline & II & III & IV & & & \\
\hline & $n=68$ & $n=62$ & $n=13$ & ANOVA & Tukey-Kontraste* & \\
\hline & $\mathrm{MW}_{ \pm} \mathrm{SD}$ & $\mathrm{MW}_{ \pm} \mathrm{SD}$ & $\mathrm{MW}_{ \pm} \mathrm{SD}$ & & II-III & II-IV \\
\hline CDI & $3,77 \pm 2,82$ & $4,92 \pm 2,53$ & $5,86 \pm 2,73$ & $p \leq 0,001$ & $p \leq 0,05$ & $p \leq 0,05$ \\
\hline \multicolumn{7}{|l|}{ SGRQ } \\
\hline Symptome & $51,95 \pm 28,17$ & $65,36 \pm 19,61$ & $64,61 \pm 17,59$ & $p \leq 0,01$ & $p \leq 0,01$ & n.s. \\
\hline Aktivitäten & $51,19 \pm 24,92$ & $68,92 \pm 17,10$ & $74,48 \pm 19,14$ & $p \leq 0,001$ & $p \leq 0,001$ & $p \leq 0,001$ \\
\hline Beeinträchtigung & $43,22 \pm 32,99$ & $58,40 \pm 28,15$ & $69,28 \pm 32,93$ & $\mathrm{p} \leq 0,01$ & $\mathrm{p} \leq 0,05$ & $p \leq 0,05$ \\
\hline Gesamt & $47,09 \pm 27,43$ & $62,74 \pm 20,93$ & $70,08 \pm 23,93$ & $p \leq 0,001$ & $p \leq 0,01$ & $p \leq 0,01$ \\
\hline
\end{tabular}

MW: Mittelwert; SD: Standardabweichung; CDI: COPD-Disability-Index, SGRQ: St. George's Respiratory Questionnaire

Statistische Verfahren: ANOVA = Univariate Varianzanalysen sowie Tukey-post-hoc-Tests für gezielte Gruppenvergleiche.

${ }^{*}$ Keine signifikanten Mittelwertsunterschiede zwischen den Stadien III und IV

nach der Beeinträchtigung durch Atemnot auffassen, sondern möglicherweise die Gesamtbeeinträchtigung der Atmung, d.h. durch Husten, Atemgeräusche, Kurzatmigkeit, Atemnot etc. beurteilen.

Die hohen internen Konsistenzen in der Selbstbeurteilungs- und gleichermaßen in der Fremdbeurteilungsversion sind Belege für die gute Reliabilität des Verfahrens. Es wird sogar trotz der Kürze des Verfahrens die für die Einzelfalldiagnostik wünschenswerte Marke von über 0.85 [39] erreicht.

Die Validität ist als sehr zufriedenstellend zu bewerten. Die Richtung und Höhe der Korrelationen mit konstruktverwandten Skalen spricht für die konvergente Validität. Erwartungsgemäß zeigen sich die höchsten Korrelationen mit dem Fragebogen zur krankheitsspezifischen Lebensqualität (SGRQ-Gesamt $r=0.83$ ). Ebenfalls hoch $(r=0.69)$ ist die Korrelation zur Fremdeinschätzung der COPD-bedingten Beeinträchtigung durch die Angehörigen, so dass davon ausgegangen werden kann, dass Ehepartner die COPD-bedingte Beeinträchtigung der Patienten gut einschätzen können.

Die COPD-bedingte Beeinträchtigung korreliert wie der SGRQ (und der PDI bei Schmerzpatienten [25]) positiv mit „Ängstlichkeit“ und „Depressivität“. Die mittlere Höhe der Korrelation ist jedoch ein Beleg dafür, dass der CDI mit der COPD-bedingten Beeinträchtigung mehr erfasst als nur Ängstlichkeit oder Depressivität. Die Korrelationen zwischen dem CDI als Selbstbeurteilungsfragebogen und den funktionellen Parametern ( $\mathrm{FEV}_{1}$, Gehstrecke) sind ebenfalls signifikant, fallen aber durch die Methodenvarianz (Selbstbeurteilungsfragebogen vs. physiologische Daten) erwartungsgemäß niedriger aus [14].

Die Höhe der Korrelation zwischen $\mathrm{FEV}_{1}$-Werten und $\mathrm{CDI}$ in der vorliegenden Studie $(\mathrm{r}=-0.26, \mathrm{p} \leq 0,01) \quad$ bzw. zwischen $\mathrm{FEV}_{1}$-Werten und SGRQ ist absolut vergleichbar [6,40-42]. Darüber hinaus unterscheiden sich die CDI-Werte in Abhängigkeit vom COPD-Stadium. Diese Stadienabhängigkeit ist auch für den SGRQ trotz z.T. divergierender Schweregradeinteilung wiederholt bestätigt worden ([43,44] vs. GOLD-Stadien [45-47], ausgenommen [48]). Unklarheit besteht aber darüber, ob es zwischen den GOLD-Stadien II-IV hinsichtlich der krankheitsspezifischen Lebensqualität unterschiedlich große Differenzen gibt. So berichten 2 Studien eine größere Differenz zwischen den Stadien III und IV im Vergleich zu den Stadien II und III [45,47]. Eine andere Studie zeigt dagegen einen signifikanten Unterschied zwischen den Stadien II und III, nicht aber zwischen III und IV - obwohl die absoluten Mittelwertsdifferenzen zwischen den Stadien II und III bzw. III und IV gleich groß sind [46]. Bei Paarvergleichen zwischen aufeinander folgenden GOLD-Stadien lassen sich - wie beim CDI in der vorliegenden Studie - auch beim SGRQ oft keine signifikanten Unterschiede nachweisen. Das liegt zum einen an der oft ungleichen Zellenbesetzung (meist deutlich weniger COPD-Patienten Stadium IV) und zum anderen an der erheblichen Variabilität der SGRQ-Werte innerhalb der COPD-Patienten ein und des selben Schweregrads.

Der Geschlechtereffekt, dass Frauen im CDI weniger COPD-bedingte Beeinträchtigung als Männer in dieser Studie angeben, muss aufgrund des geringen Frauenanteils zunächst mit Vorsicht betrachtet werden. Jedoch wurde dieser Geschlechterunterschied auch bei Schmerzpatienten für den PDI bereits berichtet [25]. Die Frauen geben in der vorliegenden Studie bei allen Items weniger Beeinträchtigung an. Die höchste mittlere Differenz zeigt sich bei der COPD-bedingten Beeinträchtigung des Sexuallebens. Das bestätigt die Relevanz des Themas [49,50] - zumindest für die Männer. Wenn dieser Geschlechtereffekt sich replizieren lässt, sollten geschlechterbezogene Normwerte entwickelt werden.

Nicht unerwähnt soll die COPD-bedingte Beeinträchtigung der Angehörigen in dieser Studie bleiben, wie sie bereits auch in anderen Studien beschrieben wurde [51 - 54]. Die Angehörigen von COPD-Patienten weisen im Vergleich zur Allgemeinbevölkerung ein erhöhtes Risiko für das Auftreten psychischer Störungen auf [55] und die psychosoziale Beeinträchtigung der Angehörigen durch die Erkrankung beeinflusst wiederum die krankheitsspezifische Lebensqualität der COPD-Patienten selbst [55].

Die Generalisierbarkeit der Ergebnisse der vorliegenden Studie wird jedoch durch folgende Faktoren eingeschränkt: Zum einen handelt es sich um eine Stichprobe ambulanter, stabiler COPDPatienten. Es können daher keine Aussagen gemacht werden, inwieweit sich die Ergebnisse von denen stationärer oder exazerbierter COPD-Patienten unterscheiden. Vor dem Einsatz des CDI bei exazerbierten Patienten sollte in einer weiteren Studie die Retest-Reliabilität des CDI im stabilen Zustand der Patienten untersucht werden. Zudem sind in der vorliegenden Studie deutlich mehr Männer (78,3\% vs. 21,7\%) als Frauen unter den Patienten. In der Gruppe der über 70-jährigen ist das Geschlechterverhältnis jedoch auch in der deutschen Allgemeinbevölkerung deutlich verschoben. 19\% der Männer haben eine COPD GOLD Stadium II oder höher, aber nur 6\% der Frauen [1]. Es bleibt offen, wodurch das Geschlechterverhältnis beeinflusst wurde, da alle Patienten konsekutiv in die Studie aufgenommen wurden. Frauen lehnten eine Studienteilnahme auch nicht öfter ab als die Männer.

Die ausgezeichnete Reliabilität, die Eindimensionalität und die gute konvergente Validität, insbesondere die hohe Korrelation 
Tab. 6 COPD-Disability-Index (CDI).

Bitte geben Sie an, wie stark Sie durch Ihre Atembeschwerden in den verschiedenen Bereichen Ihres Lebens beeinträchtigt sind. Das heißt: Wie sehr hindern Ihre Atembeschwerden Sie daran, ein normales Leben zu führen? Kreuzen Sie bitte für jeden der 7 Lebensbereiche die Zahl an, die für Sie die typische Stärke der Beeinträchtigung durch Ihre Atembeschwerden beschreibt. Ein Wert von 0 bedeutet dabei überhaupt keine Beeinträchtigung, ein Wert von 10 gibt an, dass Sie in diesem Bereich durch Ihre Atembeschwerden völlig beeinträchtigt sind.

1. Familiäre und häusliche Verpflichtungen (dieser Bereich bezieht sich auf Tätigkeiten, die das Zuhause oder die Familie betreffen. Er umfasst Hausarbeit und Tätigkeiten rund um das Haus bzw. die Wohnung, auch Gartenarbeiten)
[0]
[1]
[2]
[3]
[4]
[5]
[6]
[7]
[8]
[9]
[10]
Beeinträchtigung
2. Erholung (dieser Bereich umfasst Hobbys, Sport und Freizeitaktivitäten)
[0]
[1]
[2]
[3]
[4]
[5]
[6]
[7]
[8]
[9]
Beeinträchtigung
Beeinträchtigung
[10] völlige
Beeinträchtigung

3. Soziale Aktivitäten (dieser Bereich bezie
Essen gehen und andere soziale Aktivitäten)
[0]
[1]
[2]
[3]
[4]
[5]

[6]

Beeinträchtigung

4. Beruf (dieser Bereich bezieht sich auf berufliche Aktivitäten, die ein Teil unbezahlte Arbeiten wie Hausfrauen- oder Hausmännertätigkeit ein)
[0]
[1]
[2]
[3]
[4]
[5]
[6]
keeine
5. Sexualleben (dieser Bereich bezieht sich auf die Häufigkeit und die Qualität des Sexuallebens)
[0]
[1]
[2]
[3]
[4]
[5]
[6]
[7]
[8]
[9]
Beeinträchtigung
Beeinträchtigung
6. Selbstversorgung (dieser Bereich umfasst Aktivitäten, die Selbststän
anziehen, Auto fahren, ohne dabei auf fremde Hilfe angewiesen zu sein)
[0]
[1]
[2]
[3]
[4]
[5]
[6] [7]
[7] [8]
8] [9]
[9] [10]

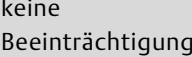
7. Atmung (dieser Bereich umfasst alle Probleme mit der Atmung)
[0]
[1]
[2]
[3]
[4]
[5]
[6]
[7]
[8]
[9]
Beeinträchtigung
Beeinträchtigung

von 0.83 zwischen dem wesentlich kürzeren COPD-DisabilityIndex (CDI) und dem SGRQ belegen, dass es sich bei dem neuen Verfahren um eine gewinnbringende Alternative zur Erfassung der COPD-bedingten Beeinträchtigung handelt. Der CDI eignet sich dank seiner Einfachheit in der Durchführung und Auswertung sowohl für den Einsatz in der pneumologischen Praxis als auch in der Forschung. Inwieweit dieses Instrument auch für den Einsatz in Interventionsstudien geeignet ist, müssen nachfolgende Untersuchungen zu weiteren Testgütekriterien (z.B. Test-Retestreliabilität, Änderungssensitivität) klären. Vor der Festlegung eines Cut-off-Wertes bzw. ggf. auch geschlechterbezogener Normwerte sind weitere Untersuchungen an anderen Stichproben nötig ( $\bullet$ Tab.6).

\section{Literatur}

1 Buist AS, McBurnie MA, Vollmer WM et al. International variation in the prevalence of COPD (The BOLD Study): a population-based prevalence study. Lancet 2007; 370: $741-750$

2 Fabel H, Konietzko N. Weißbuch Lunge 2005. Stuttgart: Thieme, 2005

3 Lopez AD, Murray CJ. The global burden of disease, 1990-2020. Nat Med 1998; 4: 1241 - 1243

4 Wijkstra PJ, Tenvergert EM, Altena $R$ van et al. Reliability and validity of the Chronic Respiratory Questionnaire (CRQ). Thorax 1994; 49: 465 467

5 Karpinski N, Petermann F, Schultz K. Kann man aus dem SGRQ (Saint George's Respiratory Questionnaire) Indikatorwerte zur Patienteneinschätzung des Reha-Erfolgs ableiten? Gesundheitswesen 2007; 69: $686-693$
6 Miravitlles M, Molina J, Naberan K et al. Factors determining the quality of life of patients with COPD in primary care. Therapeutic Advances in Respiratory Disease 2007; 1: 85 - 92

7 Guyatt GH, Berman LB, Townsend $M$ et al. A measure of quality of life for clinical trials in chronic lung disease. Thorax 1987; 42: 773 - 778

8 Puhan MA, Behnke M, Laschke $M$ et al. Self-administration and standardisation of the chronic respiratory questionnaire: a randomised trial in three German-speaking countries. Respir Med 2004; 98 : $342-350$

9 Jones PW, Quirk FH, Baveystock CM. The St George's Respiratory Questionnaire. Respir Med 1991; 85: 25 - 31

10 Hütter BO. SGRQ - St. George's Respiratory Questionnaire. In: Schumacher J, Klaiberg A, Brähler E (Hrsg). Diagnostische Verfahren zu Lebensqualität und Wohlbefinden. Göttingen: Hogrefe, 2003: 280-285

11 Rutten van Mölken M, Roos B, Nord JA van. An empirical comparison of the St George's Respiratory Questionnaire (SGRQ) and the Chronic Respiratory Disease Questionnaire (CRQ) in a clinical trial setting. Thorax 1999; 54: 995 - 1003

12 Puhan MA, Guyatt GH, Goldstein R et al. Relative responsiveness of the Chronic Respiratory Questionnaire, St. George's Respiratory Questionnaire and four other health-related quality if life instruments for patients with chronic lung disease. Respir Med 2007; 101: 308 - 316

13 Jankisz E, Moosbrugger $H$. Planung und Entwicklung von psychologischen Tests und Fragebogen. In: Moosbrugger H, Kelava A (Hrsg). Testtheorie und Fragebogenkonstruktion. Heidelberg: Springer, 2007: 27 72

14 Bühner M. Einführung in die Test- und Fragebogenkonstruktion. München: Pearson Studium, 2006

15 Moosbrugger H, Kelava A. Qualitätsanforderungen an einen psychologischen Test (Testgütekriterien). In: Moosbrugger H, Kelava A (Hrsg). 
Testtheorie und Fragebogenkonstruktion. Heidelberg: Springer, 2007: $7-26$

16 Zheng GQ Wang Y, Wang XT. Chronic hypoxia-hypercapnia influences cognitive function: A possible new model of cognitive dysfunction in chronic obstructive pulmonary disease. Med Hypotheses 2008; 71: $111-113$

17 Rourke SB, Adams KM. The neuropsychological correlates of acute and chronic hypoxemia. In: Grant I (ed). Neuropsychological assessment of neuropsychiatric disorders. New York: Oxford University Press, 1996: $379-402$

18 Crews WD, Jefferson AL, Bolduc Tet al. Neuropsychological dysfunction in patients suffering from end-stage chronic obstructive pulmonary disease. Arch Clin Neuropsychol 2001; 16: 643-652

19 Hynninen KMJ, Breitve MH, Wiborg AB et al. Psychological characteristics of patients with chronic obstructive pulmonary disease: a review. J Psychosom Res 2005; 59: 429-443

20 Pollard CA. The relationship of family environment to chronic pain disability. Dissertation Abstracts International Vol 42 (5-B), 1981

21 Dillmann $U$, Nilges $P$, Saille P et al. Behinderungseinschätzung bei chronischen Schmerzpatienten (PDI). Schmerz 1994; 8: 100-114

22 Nilges $P$, Kröner-Herwig $B$, Denecke $H$ et al. Qualitätssicherung in der Therapie chronischen Schmerzes. Schmerz 1995; 9: 242 - 247

23 Tait RC, Pollard CA, Margolis RB et al. The Pain Disability Index: Psychometric and validity data. Arch Phys Med Rehabil 1987; 68: 438 441

24 Tait RC, Chibnall JT, Krause S. The Pain Disability Index: psychometric properties. Pain 1990; 40: 171 - 182

25 Chibnall JT, Tait RC. The Pain Disability Index: Factor structure and normative data. Arch Phys Med Rehabil 1994; 75: 1082 - 1086

26 Tait RC, Chibnall JT. Factor structure of the Pain Disability Index in workers' compensation claimants with low back injuries. Arch Phys Med Rehabil 2005; 86: 1141 - 1146

27 Nagel B, Gerbershagen HU, Lindena $G$ et al. Entwicklung und empirische Überprüfung des Deutschen Schmerzfragebogens der DGSS. Schmerz 2002; 16: $263-270$

28 Singh SJ, Morgan MDL, Sciott S et al. Development of a shuttle walking test of disability in patients with chronic airways obstruction. Thorax 1992; 47: 1019-1024

29 Dyer CAE, Singh SJ, Stockley RA et al. The incremental shuttle walking test in elderly people with chronic airflow limitation. Thorax 2002; 57: $34-38$

30 Petermann F. Asthma bronchiale. Göttingen: Hogrefe, 1999

31 Schulenburg J-M Graf von der, Claes C, Greiner $W$ et al. Die deutsche Version des EuroQoL-Fragebogens. Z Gesundheitswissenschaften 1998; 6: 3-20

32 Fydrich T, Sommer G, Brähler E. Fragebogen zur Sozialen Unterstützung (F-SozU). Göttingen: Hogrefe, 2007

33 Franke GH. Symptom-Checkliste von L.R. Derogatis (SCL-90-R). Göttingen: Beltz, 2000

34 Hahlweg K. Fragebogen zur Partnerschaftsdiagnostik (FPD). Göttingen: Hogrefe, 1996

35 Weise G. Psychologische Leistungstests. Göttingen: Hogrefe, 1975

36 Jerome A, Gross RT. Pain Disability Index: Construct and Discriminant Validity. Arch Phys Med Rehabil 1991; 72: 920 - 922

37 Grönblad M, Hupli $M$, Wennerstrand $P$ et al. Intercorrelation and testretest reliability of the Pain Disability Index (PDI) and the Oswestry
Disability Questionnaire (ODQ) and their correlation with pain intensity in low back pain patients. Clin J Pain 1993; 9: 189-195

38 Grönblad M, Järvinen E, Hurri $H$ et al. Relationship of the Pain Disability Index (PDI) and the Oswestry Disability Questionnaire (ODQ) with three dynamic physical tests in a group of patients with chronic low back and leg pain. Clin J Pain 1994; 10: 197-203

39 Moosbrugger H, Rauch W. Testtheorie (Klassische Testtheorie und Items-Respone-Theorie). In: Westhoff K, Hellfritsch LJ, Hornke LF et al (Hrsg). Grundwissen für die berufsbezogene Eignungsbeurteilung nach Din 33 430. Lengerich: Pabst, 2005: 173-185

40 Ferrer M, Alonso J, Morera J et al. Chronic obstructive pulmonary disease stage and health-related quality of life. Ann Intern Med 1997; 127: $1072-1079$

41 Peruzza S, Sergi G, Vianello A et al. Chronic obstructive pulmonary disease (COPD) in elderly subjects: impact on functional status and quality of life. Respir Med 2003; 97: 612 -617

42 Haave E, Hyland ME, Skumlien S. The relation between measures of health status and quality of life in COPD. Chron Respir Dis 2006; 3: $195-199$

43 Hajiro T, Nishimura K, Tsukino $M$ et al. A comparison of the level of dyspnea vs disease severity in indicating the health-related quality of life of patients with COPD. Chest 1999; 116: 1632 - 1637

44 Hajiro T, Nishimura K, Tsukino $M$ et al. Stages of disease severity and factors that affect the health status of patients with chronic obstructive pulmonary disease. Respir Med 2000; 94: 841 - 846

45 Rutten van Mölken MPMH, Oostenbrink JB, Tashkin DP et al. Does quality of life of COPD patients as measured by the generic EuroQol Five-Dimension Questionnaire differentiate between COPD severity stages? Chest 2006; 130: 1117 - 1128

46 Antonelli-Incalzi $R$, Imperiale $C$, Catalano $F$ et al. Do GOLD stages of COPD severity really corrrespond to differences in health status? Eur Respir J 2003; 22: 444- 449

47 Ståhl E, Lindberg A, Jansson S-A et al. Health-related quality of life is related to COPD disease severity. Health Qual Life Outcomes 2005; 3 (56): $1-8$

48 Franciosi LG, Page CP, Celli BR et al. Markers of disease severity in chronic obstructive pulmonary disease. Pulmonary Pharmakology \& Therapeutics 2006; 19: 189-199

49 Schönhofer B. Sexualität bei Patienten mit beeinträchtigter Atmung. Med Klinik 2002; 97: $344-349$

50 Kaptein AA, Klink RCJ van, Kok F de. Sexuality in patients with asthma and COPD. Respir Med 2008; 102: $198-204$

51 Keele-Card G, Foxall MJ, Barron CR. Loneliness, depression, and social support of patients with COPD and their spouses. Public Health Nurs 1993; 10: $245-251$

52 Kara M, Mirici A. Loneliness, depression, and social support of Turkish patients with chronic obstructive pulmonary disease and their spouses. J Nurs Scholarsh 2004; 36: 331 - 336

53 Kanervisto M, Kaistila T, Paavilainen E. Severe chronic obstructive pulmonary disease in a family's everyday life in Finland: Perceptions of people with chronic obstructive pulmonary disease and their spouses. Nurs Health Sci 2007; 9: 40-47

54 Pinto RA, Holanda MA, Medeiros MMC et al. Assessment of the burden of caregiving for patients with chronic obstructive pulmonary disease. Respir Med 2007; 101: $2402-2408$

55 Kühl K, Schürmann W, Rief W. Mental disorders and quality of life in COPD patients and their spouses. Int J COPD in press 2008 